\title{
Visual Servoing of Space Robot for Autonomous Satellite Capture
}

\author{
By Noriyasu Inaba, ${ }^{1)}$ Mitsushige OdA ${ }^{1)}$ and Masato HaYashi ${ }^{2)}$ \\ 1) Japan Aerospace Exploration Agency, Tsukuba, Japan \\ ${ }^{2)}$ NEC TOSHIBA Space Systems, Ltd., Yokohama, Japan \\ (Received October 16th, 2002)
}

\begin{abstract}
On-orbit servicing, such as refueling, repairing, and orbit recovery, will be essential for space activities in the next generation for both manned and unmanned space systems. One of the most important and most difficult tasks in on-orbit servicing is capturing a "customer satellite" using a manipulator that can move dynamically in a wide range of space. A visual servoing technique that controls and guides the manipulator based on a camera image is required to perform this dynamic task. It is necessary to establish boundary conditions; in other words, to specify the task by assessing the environment and setting proper conditions for in order to execute it under the constraints of on-board computing power and the severe lighting conditions of space. This paper describes the design concept of a visual servoing system for a space robot and presents the results of an on-orbit experiment using Japanese Engineering Test Satellite VII (ETSVII) that was designed based on this concept.
\end{abstract}

Key Words: Space Robot, On-Orbit Servicing, Satellite Capture

\section{Introduction}

On-orbit servicing, such as refueling, repairing and orbit recovery will be essential for space activities. The first step in providing an on-orbit service is to approach and dock to a target satellite or a "customer." Manipulator capture and berthing is widely used in many space shuttle missions. ${ }^{1)}$ On these missions, the manipulator is manually controlled by an astronaut. The communication time delay between a robot satellite in low Earth orbit and a ground station is generally several seconds using a data relay satellite in geostationary orbit. This communication time delay tends to destabilize dynamic control systems that use closed loops including the ground system. Although a free-flying polyhedron was captured using a control loop including a ground system in an intravehicular experiment using the shuttle, the conditions were well designed in terms of lighting and disturbance forces acting on the object captured in the experiment. ${ }^{2)}$ Therefore, on-board visual feedback for manipulator control (i.e., visual servoing) will be essential for performing this capturing task automatically.

Studies to use visual feedback control in order to increase the positioning accuracy of robots began in the $1970 \mathrm{~s}^{3)}$ and visual servoing was proposed in the late 1980s. ${ }^{4)}$ Unlike conventional static use of vision in controlling a robot (i.e., "looking then moving and looking again"), in visual servoing, a robot is controlled continuously based on visual information. As the computing power of microprocessors improves, the capability of visual servoing is also improving in terms of tracking speed, complexity of image to be handled, and system robustness. ${ }^{5)}$

In a satellite capturing problem area, the generalized Ja-

(C) 2003 The Japan Society for Aeronautical and Space Sciences cobian matrix was proposed to control an end tip of a space manipulator as planned by taking reaction motion into account. ${ }^{6)}$ Stability analysis was conducted to use a conventional Jacobian matrix to capture a floating object with a satellite-mounted manipulator. ${ }^{7)}$ Although the knowledge obtained in the above theoretical studies is significant, a concrete control method using visual information has not been addressed. Studies based on ground experiments have also been reported. ${ }^{8-10)}$ However, synthetic technologies to design a system that works in the real space environment remained to be developed. In particular, the following three major issues should be taken into account in designing a satellite capturing system using the visual servoing technique in space.

The first issue is the computing power available in space. The second issue involves aspects of the space environment, in particular the severe and rapidly changing lighting conditions. A special aspect of space robots, the very high weight ratio of load to manipulator, is the last issue. It is necessary to specify boundary conditions; in other words, to specify the task by assessing the environment and, applying proper conditions to the task objective in order to conduct the task within the above constraints. The boundary is established by setting limits in three areas in the proposed concept.

The first limitation is established in the robot environment; for instance, reducing the range of lighting conditions by choosing a time and using a proper visual marker. The second limitation is applied to a task objective; for instance, by setting velocity limits on target motion. The last limitation is to allow for the completeness of the task under the condition of adding a function for safety abortion. Appropriate and timely instructions from the ground are taken into account before setting the above limitations. In addition, careful attention is paid to ensuring the stability of the visual 


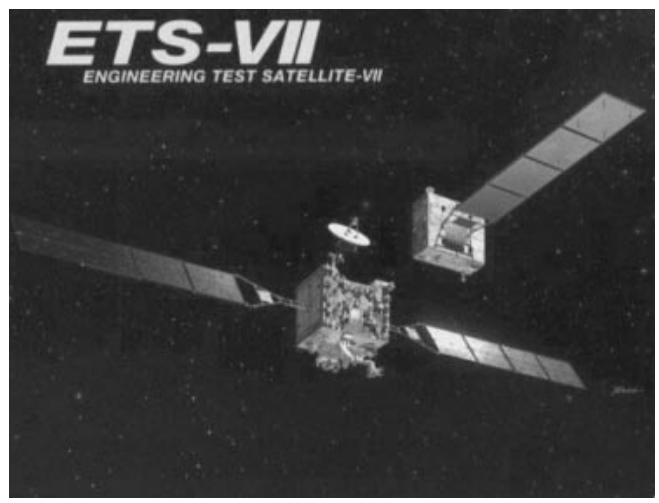

Fig. 1. ETS-VII satellites.

feedback control by considering the widely changing resonance frequency of the system.

\section{ETS-VII System}

ETS-VII is an experimental satellite for developing, and demonstrating rendezvous, docking, and space robotics technologies. ${ }^{11)}$ ETS-VII consists of two satellites, a 2.5ton chaser and a 0.4-ton target. Rendezvous and docking experiments are conducted using the two satellites, while the space robotics experiments use the robot arm on the chaser satellite. The satellites were launched together by NASDA's H-II launch vehicle on November 28, 1997 and placed into the mission orbit ( $550 \mathrm{~km}$ height and 35 -degree inclination). The designed life was 1.5 years. Figure 1 shows the ETS-VII satellites.

The manipulator (robot arm) on the chaser has six degrees-of-freedom and is $2 \mathrm{~m}$ in length. Six CCD cameras are installed to monitor dynamic experiments of the space robot and rendezvous from the ground. A hand-eye camera can also be used as an on-board vision sensor for visual servoing control of the manipulator.

\section{System Design}

Based on the concept described in the previous section, the visual servoing system for a space robot was designed using the robot system of ETS-VII. Technologies used for the system are presented in this section.

\subsection{Choice of visual marker}

The following requirements and constraints were considered when choosing a visual marker used for visual servoing of ETS-VII.

- On-board computing power

Space-qualified processors available at the time of design were capable of approximately 1 MIPS. The best image processing achieved by this computing power in real time is to identify a special visual marker and track it.

- Frequency of measurement

A measurement frequency of a few times per second is required for visual servoing to capture a quasi-static satellite whose motion is approximately up to $20 \mathrm{~mm} / \mathrm{s}$ in translation and $0.2 \mathrm{deg} / \mathrm{s}$ in rotation.
Table 1. Accuracy requirement for measurements.

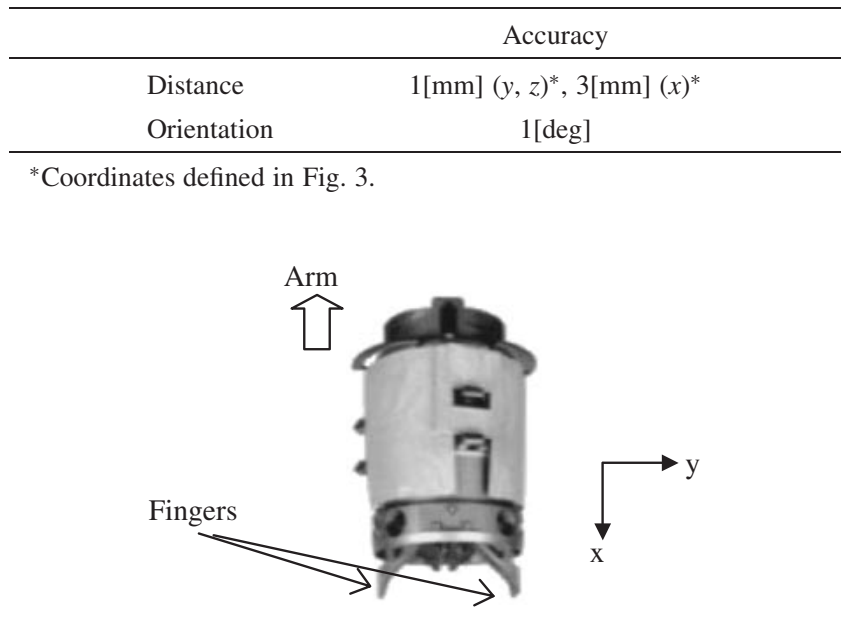

Fig. 2. End effector for satellite capture.

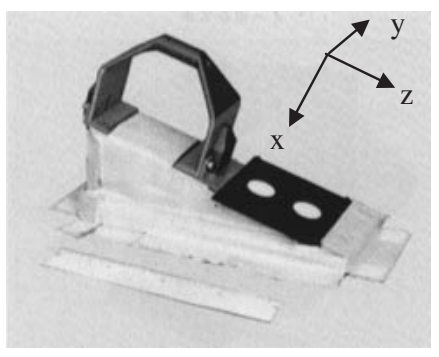

Fig. 3. Visual marker and handle.

- Range of measurement

The visual marker must be seen and recognized from target acquisition to capture where the distance between the camera and marker changes from approximately $1 \mathrm{~m}$ to $0.3 \mathrm{~m}$.

- Accuracy

The required accuracy of the measurements from a image is shown in Table 1. Since the end effector used for capturing has a wide allowance for position and orientation errors, especially in yaw angle, the yaw angle between the camera and visual marker need not be measured in this specific case. The end effector used for capturing is shown in Fig. 2.

Considering the requirements and constraints above, a visual marker consisting of two in-plane circles on a rectangle was chosen. The marker is shown in Fig. 3 together with the handle to be captured by the end effector.

\subsection{Measurements from image}

Using an image of the visual marker taken by the handeye camera, the relative position and orientation between the camera and the visual marker are measured as follows. Instructions from the ground are important for specifying the task before starting on-board measurement.

- Assistance from the ground

The first assistance from the ground is to choose a time line in order to maintain acceptable lighting conditions when the visual servoing function is used.

A satellite in low-Earth orbit circles the Earth in approximately one and a half hours. In the orbit, two light sources, the Earth and the Sun, that differ in size and lighting inten- 


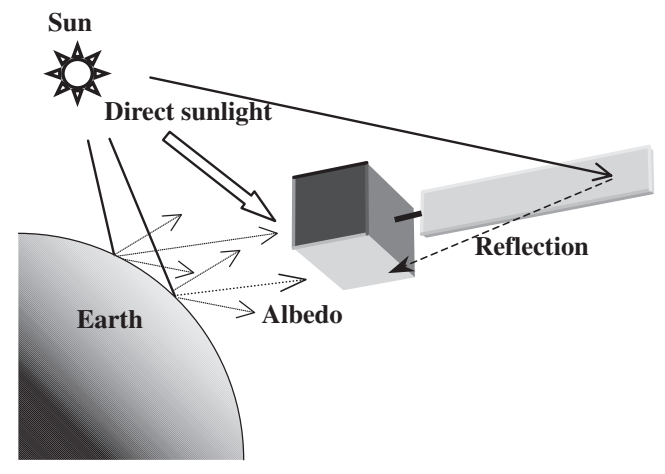

Fig. 4. Aspect of on-orbit lighting conditions.

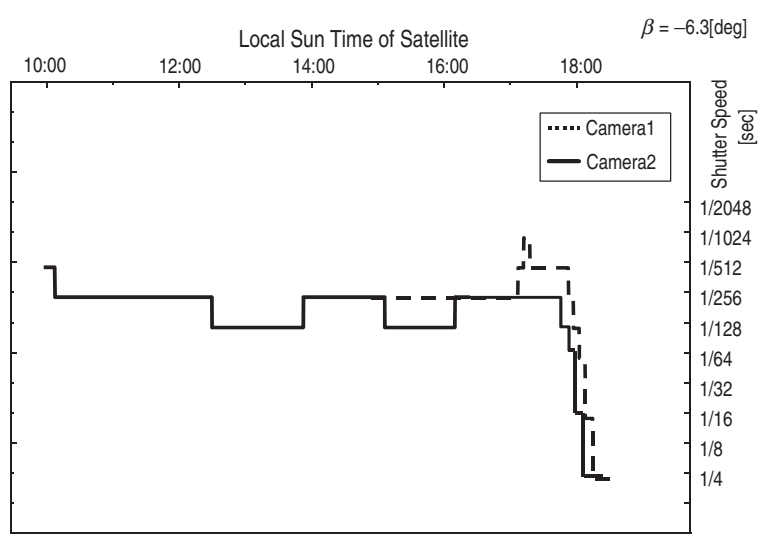

Fig. 5. Time history of optimum shutter speed.

sity illuminate the satellite. In addition, satellites sometimes go into eclipse. Thus, the lighting conditions on-orbit change rapidly and dynamically. An aspect of on-orbit lighting condition is illustrated in Fig. 4. The typical illumination factor changes from zero to several hundreds thousand lux. However, if the object is illuminated by the Earth albedo only, lighting is relatively stable for dozens of minutes. Figure 5 shows an example of optimum shutter speeds for ETSVII's on-board cameras that image the surface of a satellite illuminated by Earth albedo. A time line is designed to keep the lighting condition stable when visual servoing is used.

The second assistance is to choose a threshold for binary imaging. A threshold value for converting camera images to binary signals is instructed from the ground in order to reduce the load of the on-board processor. The threshold is determined by analyzing the histogram of an image. Figure 6 shows examples of a camera image and its histograms. We verified that the appropriate threshold can be defined widely between peaks of the histogram and holds for several minutes because of the marker design and the white surface material covering the target satellite.

On-board image processing starts after the above information is provided.

- Search process

Circles of the marker are searched spirally from the center of a binary image the first time. Once the circles and marker are found, the search starts from the point where the center of the marker existed in the previous image. In order to prevent miss-detections of the marker, the search area is limited
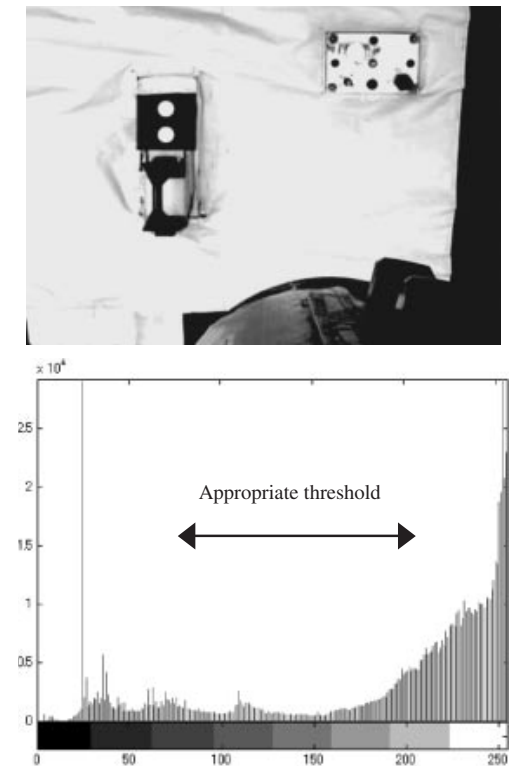

Fig. 6. Example of image and histogram.

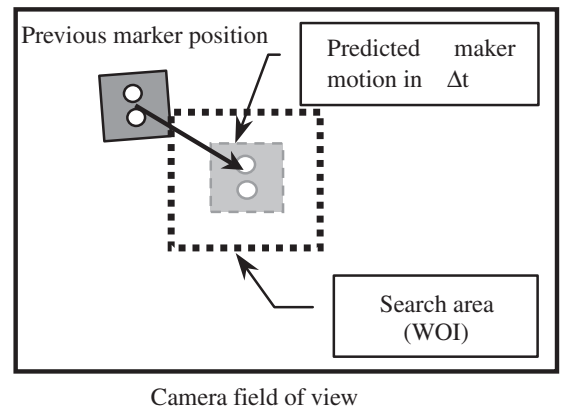

Fig. 7. Limitation of marker search area.

to inside a window of interest (WOI) that is decided from the following parameters:

- Center of marker in the previous image,

- Marker velocity relative to the camera, and

- Marker size in the image (see Fig. 7).

In the search process, a bounded white region is detected in a spiral searching line. Once the bounded white region is detected, the circumference, area, and ratio of the two parameters are measured. The bounded region is recognized as a circle of the marker if the measured parameters are within predicted values. The search process continues until the two circles of the marker are found along the searching line.

- Measurement

The measurement process follows after the two circles of the marker are recognized.

The first process extracts the following five features from an image in order to measure the relative position and orientation between the marker and the camera. The centroids of the two circles of the marker are found by detecting the centers of mass of the bounded white regions.

- Centroid of one circle $\left(y_{1}, z_{1}\right)$

- Centroid of the another circle $\left(y_{2}, z_{2}\right)$

- Area ratio of the two circles $(R)$

The observed features extracted from a image are defined as 


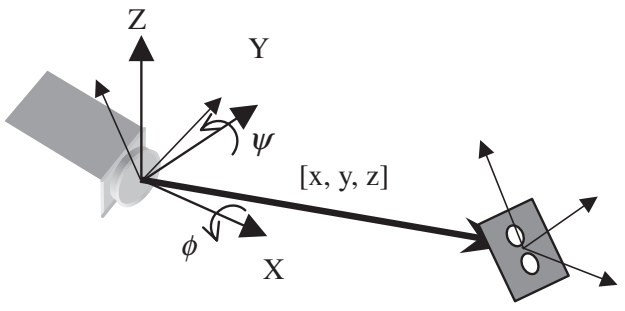

Fig. 8. Measurements from marker image.

$$
y \equiv\left[\begin{array}{lllll}
y_{1} & z_{1} & y_{2} & z_{2} & R
\end{array}\right]^{\mathrm{T}} .
$$

Since the end effector and the handle are designed to have a large capturing envelope in the yaw direction as shown in Figs. 2 and 3, it is not necessary to observe yaw attitude by marker image processing in this particular capturing task. In addition, it is well assumed that the yaw angle remains small in the necessary capturing time based on an assessment of disturbance torques attracting the target satellite on orbit. From this assumption, the relative position and orientation between the camera and the marker in the camera coordinate system are defined (Fig. 8).

$$
x_{t}^{\mathrm{C}} \equiv\left[\begin{array}{lllll}
x & y & z & \phi & \psi
\end{array}\right]^{\mathrm{T}} \text {. }
$$

The relation between the two sets of parameters are expressed as

$$
\delta y=J_{c}\left(x_{t}^{\mathrm{C}}\right) \cdot \delta x_{t}^{\mathrm{C}},
$$

where

$$
J_{c}\left(x_{t}^{\mathrm{C}}\right)=\frac{\partial y}{\partial x_{t}^{\mathrm{C}}}
$$

is a Jacobian matrix relating rate of change in pose to rate of change in feature space. Assume for the general condition that the Jacobian is square and non-singular, then the relationship

$$
\Delta x_{t}^{\mathrm{C}}=J_{c}^{-1}\left(x_{t}^{\mathrm{C}}\right) \cdot \Delta y
$$

will hold. From this relationship, the required arm tip motion in the camera frame is determined from features extracted from an image.

\subsection{Feedback control and path planning}

\section{- Timing chart}

Figure 9 shows the onboard computer's task series from image acquisition to generation of arm control commands in time sequence. Since measuring the image described in the previous paragraph takes hundreds of milliseconds, it

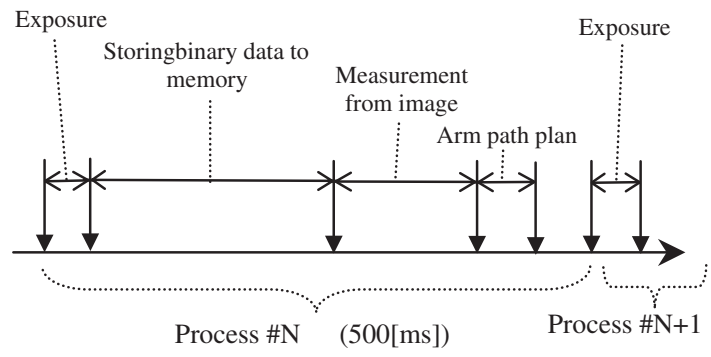

Fig. 9. Serial task process of onboard computer. is important to consider the time-delay and command discreteness of the measurements in designing the visual servoing system.

- Feed-forward control and filtering

A feed-forward control technique is used to maintain the tracking performance of the visual servoing system in spite of the latency of the image processing. Since the time required to capture the floating satellite using the visual servoing system is assumed to be within dozens of seconds, the motion of the target on a free-floating satellite is well approximated to have constant velocity motion in this case. From the marker position in camera coordinates, the marker position in the arm-base frame coordinate $\Delta x_{t}^{\mathrm{B}}$, is estimated using feed-forward techniques:

$$
\Delta x_{t}^{\mathrm{B}}=T_{\mathrm{C}}^{\mathrm{B}}(\theta)\left[\Delta x_{t}^{\mathrm{C}}+k_{\mathrm{ff}} \cdot \dot{x}_{t}^{\mathrm{C}} \cdot \delta t_{\mathrm{d}}\right],
$$

where

$T_{\mathrm{C}}^{\mathrm{B}}(\theta)$ : transformation matrix from camera coordinates to arm-base coordinates,

$\theta:$ joint angles of arm

$k_{\mathrm{ff}}$ : feed-forward gain,

$\dot{x}_{t}^{\mathrm{C}}$ : estimated target velocity in the camera coordinate, and

$\delta t_{\mathrm{d}}$ : image processing time delay (see Fig. 10).

- Path planning from discrete measurements

Maximum arm-tip speeds are established in terms of the relative velocity to the target in the camera frame as well as the absolute velocity in the arm-base frame to avoid excessive arm motion. The following two operations (translational motion and rotational motion) are conducted to keep the arm velocity within the limits when processing the velocity command using Eq. (6).

$$
\begin{aligned}
& \text { if }\left\|\Delta x_{t}^{\mathrm{C}}\right\| \geq v_{\max }^{\mathrm{C}} \cdot \Delta t \\
& \text { then } \quad \Delta x_{t}^{\mathrm{C}}=\frac{v_{\max }^{\mathrm{C}} \cdot \Delta t}{\left\|\Delta x_{t}^{\mathrm{C}}\right\|} \cdot \Delta x_{t}^{\mathrm{C}} \text { and } \\
& \text { if }\left\|\Delta x_{t}^{\mathrm{B}}\right\| \geq v_{\max }^{\mathrm{B}} \cdot \Delta t \\
& \text { then } \Delta x_{t}^{\mathrm{B}}=\frac{v_{\text {max }}^{\mathrm{B}} \cdot \Delta t}{\left\|\Delta x_{t}^{\mathrm{B}}\right\|} \cdot \Delta x_{t}^{\mathrm{B}},
\end{aligned}
$$

where

$v_{\max }^{\mathrm{C}}$ : velocity limit in camera coordinates,

$v_{\max }^{\mathrm{B}}$ : velocity limit in arm-base coordinates, and

$\Delta t$ : time interval between image processing.

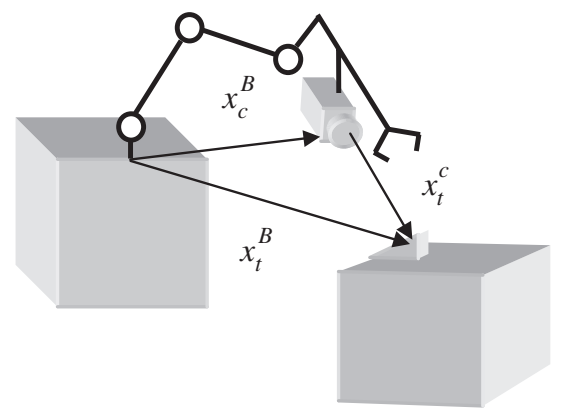

Fig. 10. Camera and arm-base coordinates. 
Finally, continuous angular velocity commands for each joint are prepared using the following equation:

$$
\Delta \theta_{\mathrm{cmd}}=J_{\theta}^{-1}(\theta) \cdot \Delta x_{t}^{\mathrm{B}}
$$

where $J_{\theta}$ is the Jacobian determined by forward kinematics depending on joint angles. The conventional Jacobian matrix employed by a ground-based manipulator is selected instead of the generalized Jacobian proposed in Ref. 6) based on control performance and computational cost. Since the mass ratio of the satellite to the manipulator is large enough, the control loop is stable as analyzed in Ref. 7).

- Control-loop stability in loaded condition

The loaded manipulator configuration should also be considered in designing the visual servoing control loop, since the control system is used until the completion of capturing phase in which the manipulator and the target satellite are joined rigidly. The cross-over frequency is set to approximately $0.1 \mathrm{~Hz}$ by using gain suppression techniques considering the need to avoid the lowest resonance frequency of the loaded manipulator. As a result, gain margins over $10 \mathrm{~dB}$ and phase margins over $40 \mathrm{deg}$ are sufficiently satisfied.

\subsection{Mode definition from approach to capture}

The sequence from approach to capture is executed automatically. Figure 11 presents the automatic mode transition of the capturing procedure. The automatic sequence is started by ground command when the visual marker is inside the camera's field of view. The sequence proceeds as follow.

\section{- Marker acquisition and holding}

Visual servoing is used to hold the marker in the center of the camera's field of view while maintaining the vertical distance between the camera and the marker. When the arm reaches a position right above the marker, the automatic se-

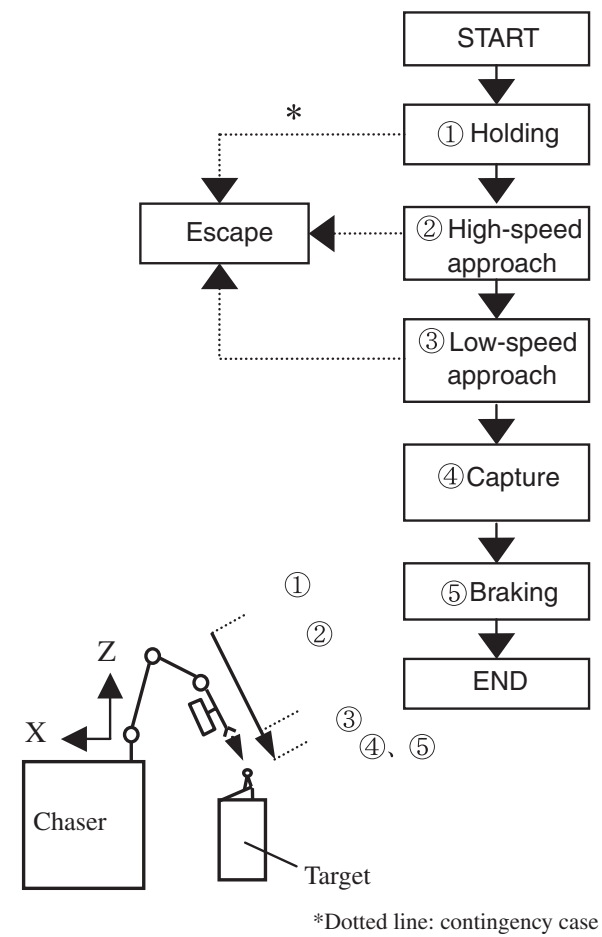

Fig. 11. Automatic mode transition. quencer enters the next mode.

- High-speed approach

The approach phase starts in high-speed mode. The armtip position and orientation are controlled to reach an intermediate point right above the grasping handle. The maximum arm-tip speed relative to the marker is limited to $10 \mathrm{~mm} / \mathrm{s}$ in this mode.

- Low-speed approach with compliance control

Once the arm reaches the intermediate point, the lowspeed approach phase starts. Compliance arm control is used by feeding-back the force-torque sensor data in order to prepare for contact. Considering the stability of the compliance control at the time of contact, the maximum arm-tip speed relative to the marker is limited to $2 \mathrm{~mm} / \mathrm{s}$ in this mode.

- Capturing

The capturing sequence starts automatically by closing the fingers of the end effector after confirming that the arm reached the targeted point relative to the marker.

- Braking

The automatic sequence ends with the braking mode. The arm stops its motion relative to the arm base with a smooth braking pattern.

\subsection{Contingency measures}

If the predetermined conditions are not satisfied in the automatic mode transition, the arm automatically exits to a predefined position where it will not contact the target satellite. The arm exits under any of the following conditions:

- Loss of visual marker,

- Departure of target from the range, or

- Trouble with the arm and/or the chaser.

Once the process enters into the capture mode, the arm will not exit even if one of the above contingencies occurs. The process continues as far as possible after the "point of no-return."

\section{Results of On-Orbit Experiments}

Two experiments were conducted to verify the visual servoing function of the ETS-VII robot satellite. The first experiment was conducted to confirm the basic functions of the visual servoing system by approaching a fixed target under on-orbit lighting conditions. A floating satellite was actually captured using the visual servoing system in the second experiment.

\subsection{Experiment for verifying basic functions}

The approach was initiated from approximately $1 \mathrm{~m}$ above the capturing position with $0.2 \mathrm{~m}$ position error in the off-axis direction. The tracking performance of the arm during the experiment is shown in Fig. 12. (The coordinate system in Fig. 11 is used.)

Control-related data used in the experiment are plotted in Fig. 13, focusing on the transition period from mode (3) to mode (4) in Fig. 11. The first data is the arm-tip positions measured from image processing data, the second is armtip position measured from arm-joint angles, and the third is arm-tip position commands issued by the controller based on image processing data. Figure 14 shows the hand-eye 


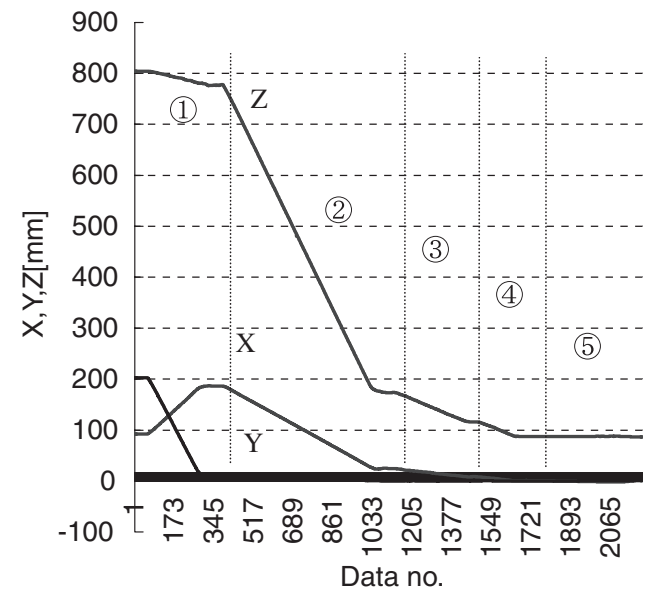

Fig. 12. Arm-tip motion profile.

1 data interval $=96.5 \mathrm{~ms}$.

Numbers in circles correspond to the mode no. in Fig. 11.

The $Z$ value at the grasping point is $95 \mathrm{~mm}$.

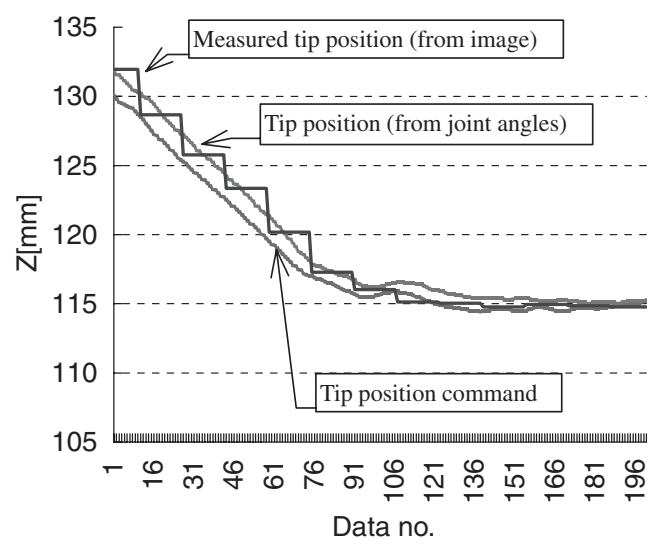

Fig. 13. Measurements and arm control performance. 1 data interval $=96.5 \mathrm{~ms}$

camera images during the experiment. Although the target satellite was fixed to the chaser satellite by a docking mechanism in the experiment, this experiment verified the basic function and performance of visual servoing in a real space environment.

\subsection{Automatic satellite capturing experiment}

An on-orbit satellite capturing experiment was conducted in September 1999. The target was released from the manipulator by gently releasing the end effector as the first step of the experiment. The target started free movement inside the open space made by the partially released docking mechanism. After separation, the manipulator again automatically approached and captured the free-floating target. The chaser attitude control was disabled from the time of release to the time of the capture in order to avoid excessive motion of the attitude control system at the time of contact (Fig. 15). The experiment was conducted successfully. ${ }^{12}$

\section{Conclusion}

The design concept for a visual servoing system to capture a free-floating satellite on orbit has been presented. Important constraints in space, such as available computing
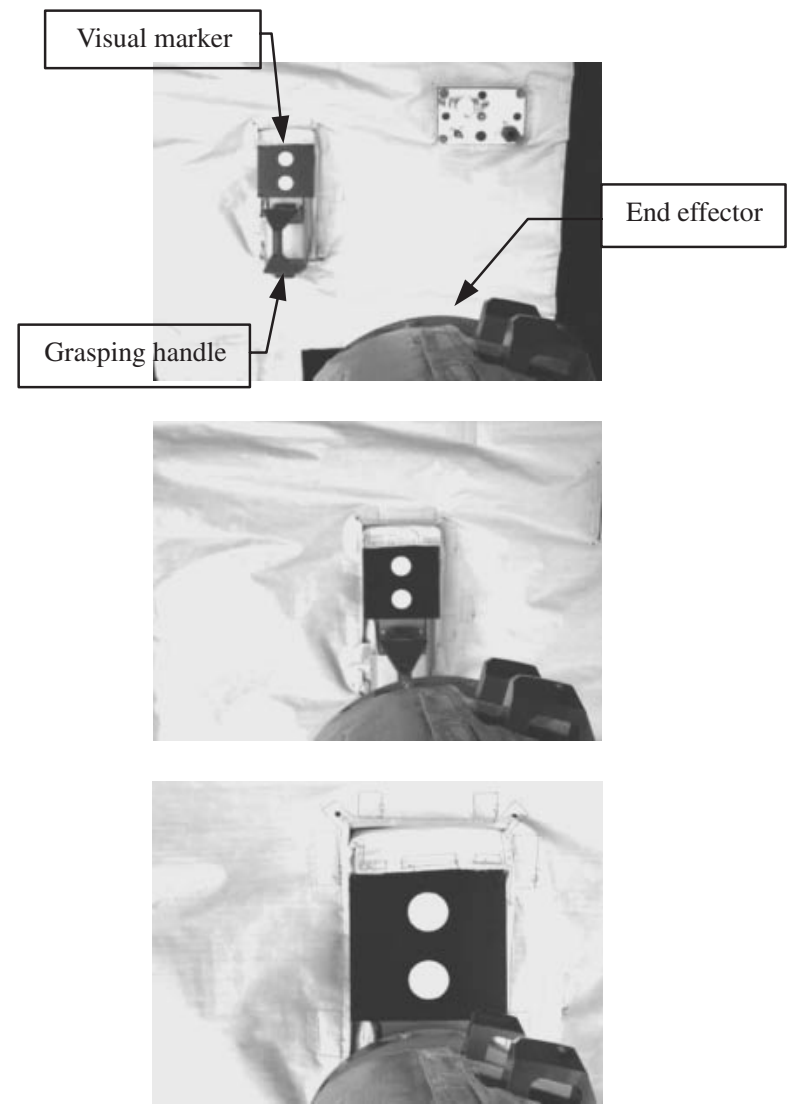

Fig. 14. Camera images during visual servoing.

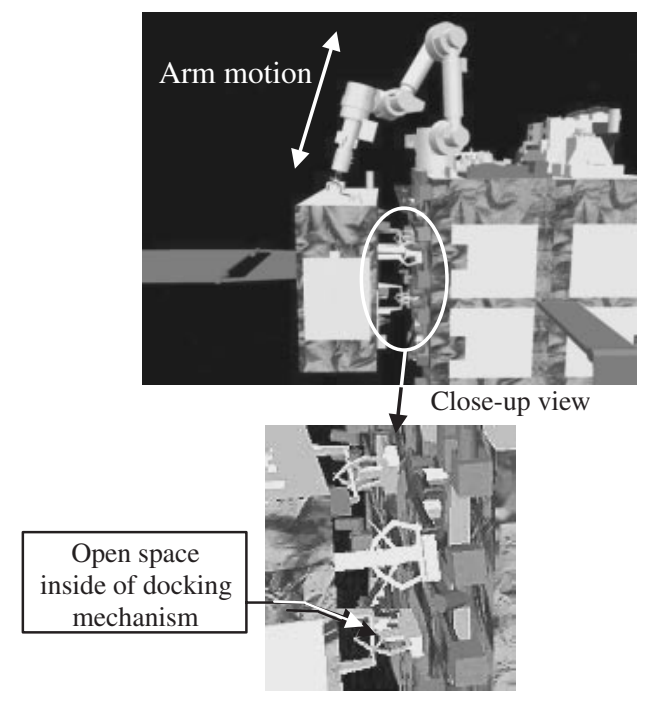

Fig. 15. Configuration of the capturing experiment.

power and severe lighting condition were taken into accounted in designing the system. In addition, the importance of setting proper boundary conditions in which the task is surely executed within the capability of the system. The validity of the concept was verified by conducting space experiments in the actual space environment using ETS-VII.

\section{References}

1) Ninomiya, K., Kawachi, M., Yamaguchi, T., Sato, M. and Tsukahara, K. : On-Orbit Operation of Free Flyer SFU's Navigation, Guidance 
and Control System, Proc. 19th Annual AAS Guidance and Control Conference, AAS96-072, 1996, pp. 583-597.

2) Skofteland, G. and Hirzinger, G.: Computing Positioning and Orientation of a Freeflying Polyhedron from 3D Data, Proc. IEEE International Conference on Robotics and Automation, 1991, pp. 150-155.

3) Shirai, Y. and Inoue, H.: Guiding a Robot by Visual Feedback in Assembling Tasks, Pattern Recognition, 5 (1973), pp. 99-108.

4) Ahlumalia, R. and Fogwell, L.: A Modular Approach to Visual Servoing, Proc. IEEE International Conference on Robotics and Automation, 1987, pp. 943-950.

5) Hashimoto, K.: Visual Servoing, World Scientific Series in Robotics and Automated Systems, Vol. 7, World Scientific, Farrer Road, Singapore, 1992.

6) Umetani, Y. and Yoshida, K.: Continuous Path Control of Space Manipulators Mounted on OMV, Acta Astronautica, 15 (1987), pp. 981986.

7) Masutani, Y., Miyazaki, F. and Arimoto, S.: Sensory Feedback Control for Space Manipulators, Proc. IEEE International Conference on Robotics and Automation, 1989, pp. 1346-1351.

8) Shimoji, H., Inoue, M., Tsuchiya, K., Ninomiya, K., Nakatani, I. and
Kawaguchi, J.: Autonomous Capture Experiment of Free-Flying Target on the Zero Gravity Simulator, Proc. International Symposium on Artificial Intelligence, Robotics and Automation in Space, 1990, pp. $77-80$.

9) Toda, Y., Iwata, T., Machida, K., Otuka, A., Toriu, H., Shinomiya, Y., Fukuda, Y., Asakura, M. and Matsuhira, N.: Development of FreeFlying Space Telerobot-Ground Experiments on Two-Dimensional Flat Testbed-, Proc. Guidance, Navigation and Control Conference AIAA, 1992, pp. 33-39.

10) Senda, K., Murotsu, Y., Mitsuya, A., Adachi, H., Shitakubo, J. and Matsumoto, T.: Hardware Experiments of a Truss Assembly by an Autonomous Space Learning Robot, J. Spacecraft Rockets, 39 (2002), pp. 267-273.

11) Oda, M., Kibe, K. and Yamagata, F.: ETS-VII — a Rendezvous Docking and Space Robot Technology Experiment Satellite, 46th International Astronautical Congress, Oslo, Norway, IAF-95-U.2.01, 1995.

12) Inaba, N. and Oda, M.: Automatic Satellite Capture by a Space Robot, Proc. IEEE International Conference on Robotic and Automation, 2000, pp. 1169-1174. 